\title{
A test of a new simulation algorithm for dynamical quarks.
}

\author{
T.D.Bakeyev \\ Moscow State University
}

\begin{abstract}
Some results of test runs on a $6^{3} \times 12$ lattice with Wilson quarks and gauge group $\mathrm{SU}(2)$ for a previously proposed fermion algorithm by A. Slavnov are presented.
\end{abstract}

\section{Introduction.}

The incorporation of fermionic degrees of freedom in lattice simulations presents a serious difficulties which stem from their being anticommuting variables. For the most actions in use which are quadratic in fermionic fields, one can eliminate fermions by an analytic integration. Unfortunately, the resulting expressions involve the determinant of a very large matrix, which induces nonlocal long-range interactions on the gauge fields, making the numerical simulations rather expensive.

The most often used method to simulate fermionic field theories is now Hybrid Monte Carlo (HMC) algorithm [1], which addresses the problem of nonlocality by linearizing the action in a succession of molecular dynamics steps. It is exact and easily implementable, but still the fermionic simulations turn out to be very time consuming and alternative approaches are welcome.

An interesting idea has been put forward by M. Lüscher [2], who proposed to approximate the inverse of the fermion matrix with a polynomial, and then interprete the determinant as the partition function of a local bosonic system. This technique has been studied thorougly for the last five years and it was claimed to be competitive to the HMC algorithm and even superior when fermions are heavy (a detailed analysis can be found in Refs [0, 10, 11]). However the efficiency of Lüescher's method is abated when quark mass decreases.

Another approach to the problem of dynamical fermions simulation was proposed by A. Slavnov in papers [6, 7], where a D-dimensional fermion determinant was presented as a path integral of a (D+1)-dimensional local bosonic action. In Ref. [9] this procedure was tested by numerical simulation of a one dimensional toy model and it was shown that correct and accurate results can be obtained with a reasonable size of lattice in auxiliary dimension. In this paper we present some results from test runs for SU(2) QCD with 2 degenerate heavy Wilson quarks, using the version of Slavnov's algorithm from Ref.[0]. 


\section{The local bosonic algorithm.}

For the local gauge theory with two flavours of Wilson quarks the effective distribution of the gauge field $U$ is given by

$$
P[U] \propto \operatorname{det}(D[U]+m)^{2} e^{-S_{g}[U]}
$$

where $D[U]$ is Wilson-Dirac operator

$$
D=\frac{1}{2} \sum_{\mu}\left[\gamma_{\mu}\left(\nabla_{\mu}+\nabla_{\mu}^{*}\right)-\nabla_{\mu}^{*} \nabla_{\mu}\right]
$$

$S_{g}[U]$ denotes the usual plaquette action and $m$ is the bare quark mass which is related to hopping parameter $k$ through $k=1 /(8+2 m)$. The lattice spacing has been set equal to unity for simplicity. It will be advantageous for us to work with hermitean operator $B=\gamma_{5}(D+m)$, so we rewrite expression (1) in the form

$$
P[U] \propto \operatorname{det} B[U]^{2} e^{-S_{g}[U]}
$$

Now following Ref.[0], we introduce five dimensional bosonic fields $\phi(x, t) \equiv \phi_{n}(x)$, where the extra coordinate $t$ is defined on one dimensional chain of the length $L$ with the lattice spacing $b$

$$
t=n b ; 0 \leq n \leq N ; L=N b
$$

and four dimensional bosonic fields $\chi(x)$. After that we present the determinant in eq.(3) in the following form

$$
\operatorname{det} B[U]^{2}=\lim _{\mu \rightarrow 0} \operatorname{det}\left(B[U]^{2}+\mu^{2}\right)=\lim _{\mu \rightarrow 0} \lim _{b \rightarrow 0 ; L \rightarrow \infty} \int e^{-S_{\mu, b, L}[U, \phi, \chi]} D \phi^{*} D \phi D \chi D \chi
$$

where $S_{\mu, b, L}$ is a local bosonic action

$$
\begin{aligned}
& S_{\mu, b, L}[U, \phi, \chi]=\sum_{x} \sum_{n=1}^{N-1}\left[-\left(\phi_{n+1}^{*}(x) \phi_{n}(x)+\text { h.c. }-2 \phi_{n}^{*}(x) \phi_{n}(x)\right)+\right. \\
& +b\left(\imath \phi_{n+1}^{*}(x) B \phi_{n}(x)+h . c\right)+b^{2} \phi_{n}^{*}(x) B^{2} \phi_{n}(x)+ \\
& \left.+b \exp \{-\mu b n\}\left(\chi^{*}(x)(\mu+\imath B) \phi_{n}(x)+\text { h.c. }\right)\right]+\frac{1}{2 \mu b} \sum_{x} \chi^{*}(x) \chi(x)
\end{aligned}
$$

and the free boundary conditions in $t$ for the fields $\phi_{n}$ are imposed

$$
\phi_{0}=0 ; \phi_{N}=0
$$

Let us prove eq.(5), which shows that lattice QCD is a limit of purely bosonic theory (see Ref.[7]). The bosonic action (6) is a linearized version of the expression in the exponent of the following integral

$$
\begin{aligned}
& I_{\mu, b, L}[U]=\int \exp \left\{\sum _ { \alpha } \sum _ { n = 1 } ^ { N - 1 } \left[\left(\phi_{n+1}^{\alpha *} \exp \left\{-\imath B_{\alpha} b\right\} \phi_{n}^{\alpha}+\text { h.c. }-2 \phi_{n}^{\alpha *} \phi_{n}^{\alpha}\right)-\right.\right. \\
& \left.\left.-b \exp \{-\mu b n\}\left(\chi^{\alpha *}\left(\mu+\imath B_{\alpha}\right) \phi_{n}^{\alpha}+h . c\right)\right]-\frac{1}{2 \mu b} \sum_{\alpha} \chi^{\alpha *} \chi^{\alpha}\right\} D \phi^{*} D \phi D \chi^{*} D \chi
\end{aligned}
$$


where instead of $x$-representation we used a basis formed by the eigenvectors of the operator $B, B_{\alpha}$ being corresponding eigenvalues. Indeed, expanding $\exp \left\{-\imath B_{\alpha} b\right\}$ in eq.(8) in a Taylor series, keeping only the terms of the order $\mathcal{O}\left(b^{2}\right)$ and substituting

$$
\frac{b^{2}}{2}\left(\phi_{n+1}^{\alpha *} B_{\alpha}^{2} \phi_{n}^{\alpha}+\text { h.c. }\right) \rightarrow b^{2} \phi_{n}^{\alpha *} B_{\alpha}^{2} \phi_{n}^{\alpha}
$$

we get the expression (6). The substitution (9) also introduces corrections of the order $\mathcal{O}\left(b^{2}\right)$ and ensures that the action (6) is strictly positive.

By changing variables

$$
\phi_{n}^{\alpha} \rightarrow \exp \left\{-\imath B_{\alpha} n b\right\} \phi_{n}^{\alpha} ; \phi_{n}^{\alpha *} \rightarrow \exp \left\{\imath B_{\alpha} n b\right\} \phi_{n}^{\alpha}
$$

we can rewrite the equation (8) as the gaussian integral over $\phi$ with the quadratic form which does not depend on $B_{\alpha}$

$$
\begin{aligned}
& I_{\mu, b, L}[U]=\int \exp \left\{\sum _ { \alpha } \sum _ { n = 1 } ^ { N - 1 } \left[\left(\phi_{n+1}^{\alpha *} \phi_{n}^{\alpha}+\text { h.c. }-2 \phi_{n}^{\alpha *} \phi_{n}^{\alpha}\right)-\right.\right. \\
& \left.\left.-b\left(\exp \left\{-\left(\mu+\imath B_{\alpha}\right) b n\right\} \chi^{\alpha *}\left(\mu+\imath B_{\alpha}\right) \phi_{n}^{\alpha}+h . c\right)\right]-\frac{1}{2 \mu b} \sum_{\alpha} \chi^{\alpha *} \chi^{\alpha}\right\} D \phi^{*} D \phi D \chi^{*} D \chi
\end{aligned}
$$

Let us integrate the expression (11) over the fields $\phi$. To do so it is sufficient to find a stationary point of the exponent. For small $b$ the sum over $n$ can be replaced by the integral over continuous variable $t=n b$ and the equations for the stationary point acquire the form

$$
\begin{aligned}
& \ddot{\phi}^{\alpha}-b^{-1} \chi^{\alpha}\left(\mu-\imath B_{\alpha}\right) e^{-\left(\mu-\imath B_{\alpha}\right) t}=0 \\
& \phi^{\alpha}(0)=\phi^{\alpha}(L)=0
\end{aligned}
$$

The replacement of the sum by the integral also introduces corrections of order $O\left(b^{2}\right)$. The solutions of these equations are:

$$
\phi^{\alpha}(t)=\frac{\chi^{\alpha}}{b\left(\mu-\imath B_{\alpha}\right)}\left(e^{-\left(\mu-\imath B_{\alpha}\right) t}+\frac{t}{L}\left(1-e^{-\left(\mu-\imath B_{\alpha}\right) L}\right)-1\right)
$$

Substituting these solutions to the integrand (11), integrating over $t$ and rescaling the fields $\chi \rightarrow \sqrt{b L} \chi$, we get

$$
\lim _{b \rightarrow 0} I_{\mu, b, L}[U]=\int \exp \left\{-\sum_{\alpha} \frac{\chi^{\alpha *} \chi^{\alpha}}{\mu^{2}+B_{\alpha}^{2}}\left(1-2 e^{-\mu L} \cos B_{\alpha} L+e^{-2 \mu L}\right)\right\} D \chi^{*} D \chi
$$

Therefore:

$$
\lim _{b \rightarrow 0, L \rightarrow \infty} I_{\mu, b, L}[U]=\operatorname{det}\left(B[U]^{2}+\mu^{2}\right)
$$

The equality (5) is proven. For finite $b, L$ and $\mu$ this equation is approximate 


$$
\operatorname{Det} B[U]^{2} \approx \int e^{-S_{\mu, b, L}[U, \phi, \chi]} D \phi^{*} D \phi D \chi^{*} D \chi
$$

and has to be corrected by the terms

$$
O\left(b^{2}\right) ; O\left(e^{-\mu L}\right) ; \mathcal{O}\left(\mu^{2}\right)
$$

It was shown that lattice QCD is a limit of a purely bosonic local theory with the action

$$
S_{e f f}[U, \phi, \chi]=S_{g}[U]+S_{\mu, b, L}[U, \phi, \chi]
$$

Making use of this action, we can simulate the theory by locally updating the boson fields $U, \phi$ and $\chi$.

Choosing the number of points in auxiliary dimension $N$ we can fully control the systematic errors of the algorithm (17). Indeed, fixing $\mu L=\epsilon$ we have

$$
N=\frac{\epsilon}{b \mu}
$$

Increasing $N$ we can increase $\epsilon$ and decrease $b$ and $\mu$, making the errors (17) small enough in comparison with an available level of statistical precision. Since the computational cost of the algorithm depends on $N$, the parameters $\epsilon, \mu, b$ should be optimally tuned to make $N$ as small as possible.

\section{Test results. Investigation of systematic errors of the algorithm.}

In this section we present the results from numerical simulations of the bosonic theory (18) on a $6^{3} \times 12$ lattice with gauge group $\mathrm{SU}(2)$ and bare parameters $\beta=2.12$ and $k=0.15$. The simulations for the same model and parameters were already done in Ref. [5] within the framework of Luescher's formulation of the dynamical quarks problem [2]. That will allow us to compare the algorithm explored in this paper with the Luescher's one.

The implementation was done on a Quadrics Q4 machine (APE computer) with 32 nodes. A full update cycle involved 1 heatbath and 1 overrelaxation sweep for the fields $\phi_{n}$ followed by 1 heatbath sweep for the fields $\chi$ and gauge fields $U$. (Our preparatory tests showed that overrelaxing the gauge fields and the fields $\chi$ does not decrease autocorrelaton times $\tau_{\text {int }}$. On the contrary, overrelaxing the fields $\phi_{n}$ can decrease $\tau_{\text {int }}$ substantially.) For each set of parameters $\mu, b, N$ we have performed at least 30000 and sometimes up to 80000 such cycles after thermalization of the system, using the random number generator of Ref.[3].

Our test runs were structurally divided into a sequence of "experiments" in which the systematic errors (17) were studied separately. In each "experiment" we fixed some of the parameters $\mu, b, \mu L$, holding the corresponding errors fixed and altered the other parameters. This way of investigation of the systematic errors allows to determine the values of parameters at which one may be able to get correct results at given level of statistics, using the bosonic theory with relatively small number of points in auxiliary dimension $N$. 


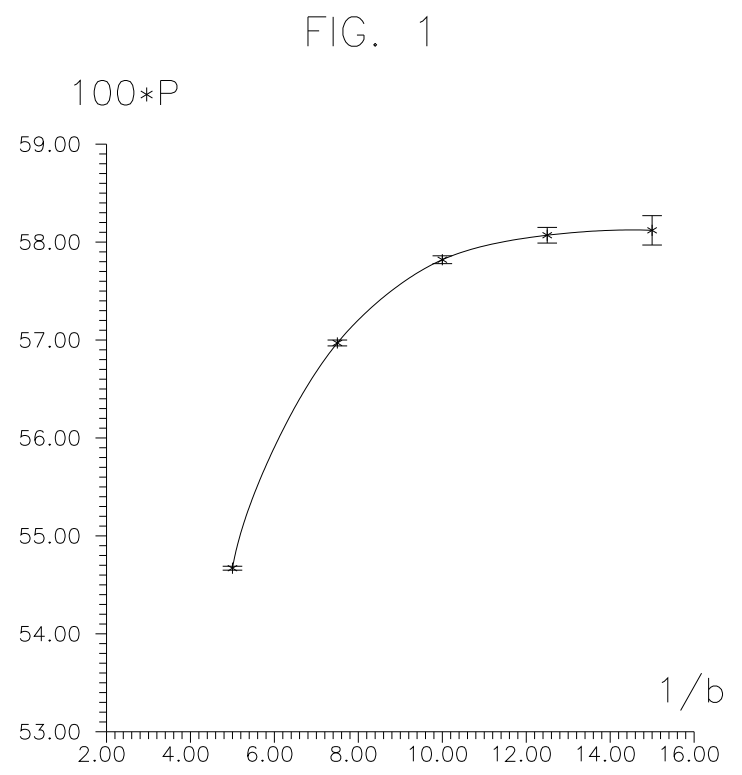

In the first "experiment" we fixed $\mu L=4.0, \mu=1.0$ to study the alteration of the plaquette value when the lattice spacing in auxiliary dimension $b$ changes. By doing so we investigated the algorithm's error of the order $\mathcal{O}\left(b^{2}\right)$ for fixed errors $\mathcal{O}\left(e^{-\mu L}\right)$ and $\mathcal{O}\left(\mu^{2}\right)$. The results of the simulations are reported in Table 1. In Fig. 1 the plaquette values multiplied by 100 are plotted against the inverse lattice spacing $b^{-1}$. The plot shows that the plaquette value stabilizes quickly when $b$ decreases. From the data obtained we see that at this level of statistics one may be able to do with $b=0.08$ to make the systematic error of the order $\mathcal{O}\left(b^{2}\right)$ small.

\begin{tabular}{|l|l|l|l|l|l|l|}
\hline $\mathrm{N}$ & 20 & 30 & 40 & 50 & 60 & $\mathrm{HMC}$ \\
\hline$b^{-1}$ & 5 & 7.5 & 10 & 12.5 & 15 & - \\
\hline plaquette & $0.5467(2)$ & $0.5697(3)$ & $0.5782(4)$ & $0.5807(8)$ & $0.5812(12)$ & $0.5796(6)$ \\
\hline$\tau_{\text {int }}$ & $24(2)$ & $67(8)$ & $94(18)$ & $*$ & $*$ & - \\
\hline
\end{tabular}

Table 1: Simulation results for the plaquette at $\mu=1.0$ and $\mu b N=4.0$. The autocorrelation time for the plaquette is measured in unit of iteration sweeps. A star means that the autocorrelation time was too long to be measured accurately. In the last column we adduce for comparison a value obtained from the Hybrid Monte Carlo for the same lattice and parameters $\beta, k$ in Ref.[5].

In the second "experiment" we attempted to investigate the character of systematic deviations of the order $\mathcal{O}\left(e^{-\mu L}\right)$. For that purpose we fixed $\mu=1$ and $b=0.1$ in order to fix the deviations connected with the errors of the order $\mathcal{O}\left(\mu^{2}\right)$ and $\mathcal{O}\left(b^{2}\right)$. As a work hypothesis we assumed that the correction terms of the order $\mathcal{O}\left(b^{2}\right)$ to the approximation (16) do not depend on $N$ at fixed $b$ (it will be seen from the simulation results that this hypothesis is wrong).

The results are reported in Table 2. In Fig. 2 the plaquette values multiplied by 100 are plotted against the number of points in auxiliary dimension $N$. 


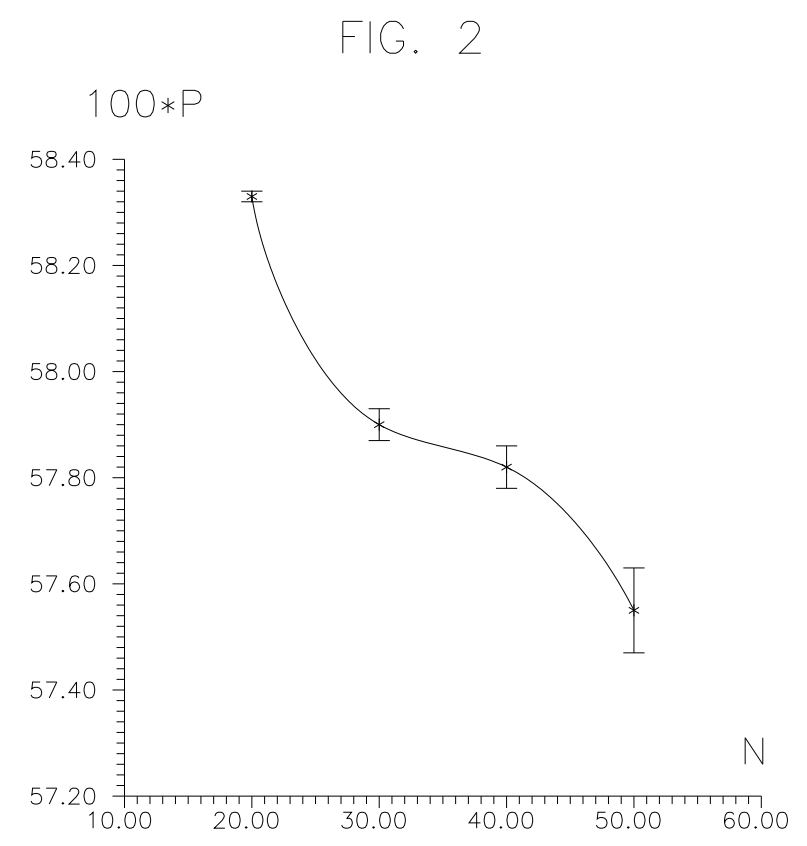

\begin{tabular}{|l|l|l|l|l|}
\hline $\mathrm{N}$ & 20 & 30 & 40 & 50 \\
\hline$\mu L$ & 2 & 3 & 4 & 5 \\
\hline plaquette & $0.5833(1)$ & $0.5790(3)$ & $0.5782(4)$ & $0.5755(8)$ \\
\hline$\tau_{\text {int }}$ & $28(2)$ & $65(8)$ & $94(18)$ & $*$ \\
\hline
\end{tabular}

Table 2: Simulation results at $\mu=1.0, b=0.1$.

The plot shows that the plaquette value does not stabilize when $N$ increases. Since the error of the order $\mathcal{O}\left(\mu^{2}\right)$ can not depend on $N$ (the approximation $\operatorname{Det} B^{2} \approx \operatorname{Det}\left(B^{2}+\mu^{2}\right)$ was used even before the bosonization procedure and introduction of auxiliary dimension), the only explanation to this result is the dependence on $N$ of the error $\mathcal{O}\left(b^{2}\right)$ for fixed $b$.

To demonstrate this on a theoretical level, let us return to the expression (11) and integrate it over $D \phi_{n}$ for finite $b$ (i.e. not using the simplifying assumption $b \rightarrow 0$ ). Then the equations for the stationary point acquire the form

$$
\begin{aligned}
& 2 \phi_{n}^{\alpha}-\phi_{n+1}^{\alpha}-\phi_{n-1}^{\alpha}+b \chi^{\alpha}\left(\mu-\imath B_{\alpha}\right) e^{-\left(\mu-\imath B_{\alpha}\right) n b}=0 \\
& \phi_{0}=\phi_{N}=0
\end{aligned}
$$

The solutions of these equations are

$$
\phi_{n}^{\alpha}=\frac{\chi^{\alpha}}{b\left(\mu-\imath B_{\alpha}\right)}\left(e^{-\left(\mu-\imath B_{\alpha}\right) n b}+\frac{n b}{L}\left(1-e^{-\left(\mu-\imath B_{\alpha}\right) N b}\right)-1\right) \frac{b^{2}\left(\mu-\imath B_{\alpha}\right)^{2}}{2 \cosh \left[\mu b-\imath b B_{\alpha}\right]-1}
$$

Substituting these solutions to the integrand (11), omiting the terms of the order $\mathcal{O}\left(e^{-\mu L}\right)$, summing over $n$, rescaling the fields $\chi \rightarrow \sqrt{b L} \chi$ and keeping only the terms of the order $\mathcal{O}\left(b^{2}\right)$, we get

$$
I[B] \approx \int \exp \left\{-\sum_{\alpha} \frac{\chi^{\alpha *} \chi^{\alpha}}{\mu^{2}+B_{\alpha}^{2}}\left[1+\frac{b^{2}\left(B_{\alpha}^{2}-\mu^{2}\right)}{6}+\frac{N b^{3}}{24 \mu}\left(3 \mu^{2}-B_{\alpha}^{2}\right)\left(B_{\alpha}^{2}+\mu^{2}\right)\right]\right\} D \chi^{*} D \chi
$$


From the expression (22) we see that the algorithm's error terms of the order $\mathcal{O}\left(b^{2}\right)$, which are associated to replacement of the sum over $n$ by the integral, explicitly depend on $N$ for fixed $b$. Hence a more careful approach to an analysis of systematic error of the order $\mathcal{O}\left(b^{2}\right)$ is needed.

As a rule for interesting cases (and for the model under consideration) the following condition holds

$$
\mu \ll\|B\|
$$

Therefore, from the expresions (14,22) one sees that the relative systematic error for measurement of arbitrary quantity in Slavnov's algorithm can be represented as follows

$$
\begin{aligned}
& \Delta=\Delta_{1}+\Delta_{2}+\Delta_{3}+\Delta_{4} \\
& \Delta_{1}=F b^{2} ; \Delta_{2}=G \frac{N b^{3}}{\mu} ; \Delta_{3}=H e^{-\mu L} ; \Delta_{4}=P \mu^{2}
\end{aligned}
$$

where the values $F, G, H, P$ are approximately constant and depend on the parameters $b, N, \mu$ only in the next order of perturbation theory.

Using the formula (24), let us implement a more correct investigation of the algorithm's deviation of the order $\mathcal{O}\left(e^{-\mu L}\right)$.

In the third "experiment" we fixed $\mu=1, \frac{b^{3} N}{\mu}=0.04\left(\Delta_{2}=\right.$ const, $\Delta_{4}=$ const $)$, and increased $N$ (decreasing the errors $\Delta_{1}$ and $\Delta_{3}$ ). The results are reported in Table 3 .

\begin{tabular}{|l|l|l|l|l|l|}
\hline$N$ & 20 & 30 & 40 & 50 & 60 \\
\hline$b$ & 0.1260 & 0.1101 & 0.1000 & 0.0928 & 0.0874 \\
\hline$\mu L$ & 2.52 & 3.30 & 4.00 & 4.62 & 5.24 \\
\hline plaquette & $0.5780(3)$ & $0.5773(6)$ & $0.5782(4)$ & $0.5773(4)$ & $0.5770(10)$ \\
\hline
\end{tabular}

Table 3: Simulation results at $\mu=1.0, \frac{b^{3} N}{\mu}=0.04$.

From Table 3 one sees that the plaquette value changes slowly when $N$ increases and it's alteration is not monotonic. Such a behavior can be explained by a mutual compensation of the errors $\Delta_{1}$ and $\Delta_{3}$. Puting together the results from tables 1 and 3 one can conclude that at given level of statistics it is enough to hold $\mu L=5$ in order to make the systematic error of the order $\mathcal{O}\left(e^{-\mu L}\right)$ small.

In the fourth "experiment" we fixed $\frac{b^{3} N}{\mu}=0.04, \mu L=3.3\left(\Delta_{2}=\right.$ const, $\Delta_{3}=$ const $)$, and decreased $\mu$ (decreasing the errors $\Delta_{1}$ and $\Delta_{4}$ ). The plaquette value and the masses of $\pi$ and $\rho$ mesons were mesured. The results of the simulations are reported in Table 4 . In Fig. 3 the plaquette values multiplied by 100 are plotted against the value of auxiliary "mass" $\mu$. 


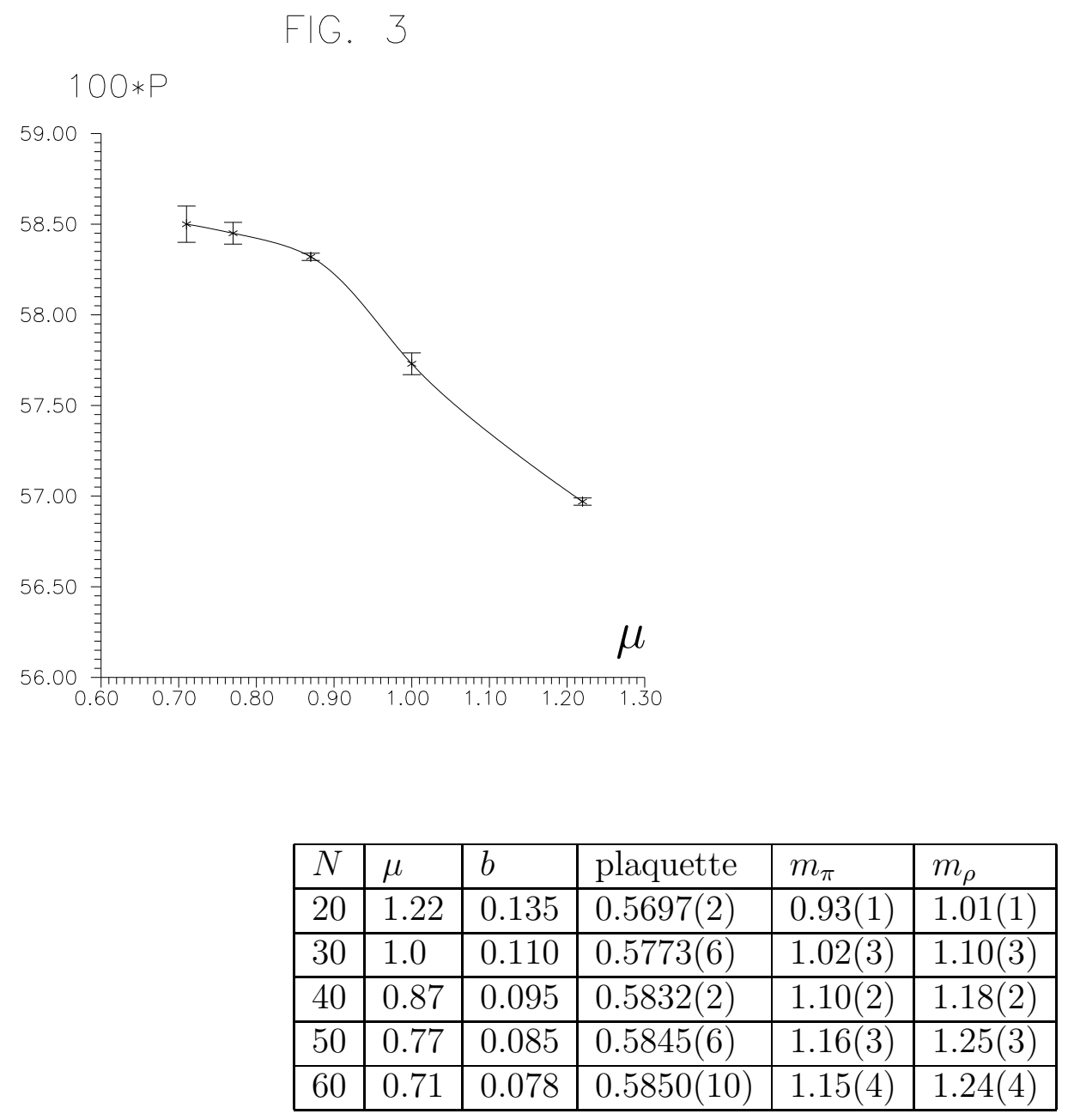

Table 4: Simulation results for the plaquette and meson masses at $\frac{b^{3} N}{\mu}=0.04, \mu b N=3.3$.

The simulation results for the plaquette and meson masses from Table 4 show that the measured values stabilize when $\mu$ decreases. From the data obtained we see that at this level of statistics one may be able to do with $\mu=0.8$ to make the error of the order $\mathcal{O}\left(\mu^{2}\right)$ small.

Let us make a conclusion to this section. We investigated the systematic errors of Slavnov's algorithm and got the values of algorithm's parameters at which in the tested model the errors can be considered small at the given level of statistical precision. These values are as follows:

$$
\frac{b^{3} N}{\mu} \leq 0.03 \quad ; \quad b \leq 0.08 \quad ; \quad \mu b N \geq 5.0 \quad ; \quad \mu \leq 0.8
$$

From the conditions (25) we infer that it is enough to take $N=100$ points in auxiliary dimension to get correct results in the tested model. To check up this statement we made a control test run at $N=100 ; \mu=0.8 ; b=0.062$. The measured plaquette value $<P>=0.5805(11)$ within the statistical error coincided with the result obtained from HMC in paper [5]: $\left\langle P>_{H M C}=0.5796(6)\right.$. 


\section{Conclusion.}

In this paper we performed the first investigation of systematic error effects of Slavnov's algorithm in a realistic model. It was shown that at this level of statistics one may be able to do with $N=100$ points in auxiliary dimension. This is comparable with the number of bosonic fields which was necessary to get the correct results in the same model from Lüscher's algorithm in Ref. [5].

Note that the action (6) and the effective bosonic action in Lüscher's formulation of the dynamical fermions problem are similar in a sense that computational effort per update cycle is almost the same for both algorithms if the number of bosonic fields in Lüscher's algorithm is equal to the number of points in auxiliary dimension $N$ in action (6) (the update of the fields $\chi$ in the action (6) takes less than 1\% of total computer time). Accordingly both algorithms are comparable in an amount of computer time which is necessary per full update cycle.

We observed the growth of autocorrelation times when $N$ increases. It seems that such an autocorrelation behavior is the general feature of multibosonic algorithms (the theoretical justification of this fact can be found in Ref. 团). The autocorrelation times in our simulations for $N=20$ and 40 are almost two times less than the ones for the same numbers of bosonic fields in Lüscher's formulation measured in Ref. [4], but our MC runs were rather short and that may tend to underestimating the algorithm's autocorrelation time. A more careful investigation of autocorrelation behaviour in Slavnov's algorithm requires further extensive tests on a more powerful computers.

It is interesting that the systematic errors $\mathcal{O}\left(b^{2}\right)$ and $\mathcal{O}\left(\mu^{2}\right)$ from the one side and $\mathcal{O}\left(e^{-\mu L}\right)$ from the other side for the measured quantities compensate each other. This fact can lead to the considerable improvement of results even for moderate values of $N$ as it was observed in the present research.

The present performance of Slavnov's algorithm can be considerably improved by diminishing the systematic error from $\mathcal{O}\left(b^{2}\right)$ to $\mathcal{O}\left(b^{4}\right)$. This can be put into effect by adding to the action (6) the terms

$$
\Delta S=\sum_{x}\left[\alpha(b, N, \mu) \chi^{*}(x) B^{2} \chi(x)+\delta(b, N, \mu) \chi^{*}(x) \chi(x)\right]
$$

where the coefficients $\alpha(b, N, \mu)$ and $\delta(b, N, \mu)$ can be computed theoretically (this work is under progress). Also even-odd preconditioning can be incorporated to reduce the number of points in auxiliary dimension $N$.

\section{Acknowledgements.}

The numerical simulations reported in this paper were done using the APE Q4 computer of von Neumann Institute of Computational Physics (Jülich, Germany). I am very grateful to administration of von Neumann Institute and especially to Prof. K. Schilling for this possibility. I am very beholden to Prof. A. Slavnov (Steclov Mathematical Institute) for continuous attention to this work and generous help. Special thanks to Prof. K. Schilling, Dr. T. Lippert and other members of von Neumann Institute of Computing/DESY for hospitality and numerous discussions.

This research was supported by INTAS-RFBR under grant 95-0681, Russian Basic Research Fund 96-01-00551, and Presidential grant for support of leading scientific schools 
96-15-96208.

\section{References}

[1] S. Duane, A.D. Kennedy, B.J. Pendelton, D. Roweth // Phys. Lett. B195 (1987) 216

[2] M.Luescher // Nucl. Phys. B418 (1994) 637

[3] M. Lüscher // Comp. Phys. Comm. 79 (1994) 100

[4] B. Jegerlehner // Nucl.Phys. Proc.Suppl. B42 (1995) 879; hep-lat 9411065

[5] B. Bunk, K. Jansen, B. Jegerlehner, M. Lusher, H. Simma, R. Sommer // Nucl Phys. Proc. Suppl. B42 (1995) 49.

[6] A.A. Slavnov // Phys. Lett. B338 (1996) 147; hep-lat 9605006

[7] A.A. Slavnov // hep-th 9611154

[8] K. Jansen // Nucl.Phys.B Proc.Suppl. 53 (1997) 127; hep-lat 9607051

[9] T. Bakeyev, A. Veselov, M. Polikarpov, A. Slavnov // Theor.Math.Phys. 113 (1997) 58; hep-lat 9702002

[10] Ph. de Forcrand // hep-lat 9702009

[11] Ph. de Forcrand // hep-lat 9903035 